\title{
Second rock from the Sun
}

\section{Andrew P. Ingersoll relishes a study of scientific discoveries on hot, toxic Venus.}

$\mathrm{V}$

enus is the closest to Earth of all the planets - in distance, size, mass and composition. But it is a hellish place, with opaque clouds of sulphuric acid, crushing atmospheric pressures and surface temperatures high enough to melt lead. Not photogenic and too blistering for conventional rovers and landers, Venus has been passed over by the big missions. Nevertheless, Venus exploration and the interpretation of the resulting data have had their fair share of exciting moments. It is this history that physicist Fredric Taylor relates in his informative primer, The Scientific Exploration of Venus.

The first hints that Venus was not like Earth came in the 1950s. Earth-based radio telescopes, peering through the planet's clouds, reported a temperature of more than $300^{\circ} \mathrm{C}$ (today the estimate is rather higher). But was that the temperature of Venus's ionosphere - the upper atmosphere - or its surface?

Astronomer Carl Sagan, whose doctoral thesis showed how the greenhouse effect could account for high surface temperatures on Venus, set out to test his theory. He lobbied the newly established NASA to build the first interplanetary spacecraft and equip it with the right instrument - a microwave radiometer. Identical spacecraft, Mariner 1 and 2, were built and launched in 1962. Mariner 1 failed, but Mariner 2 made the three-month trip to Venus and took the crucial measurement, showing that the temperature was greatest when the radiometer looked straight down at the surface and least when it looked horizontally through the ionosphere.

Taylor also tells the story of the US-Soviet race to explore Venus. Relative to the cold war, it was almost friendly, and indeed may have helped the slow easing of tensions between the two superpowers. Scientists read each others' papers and started to visit each others' countries. Mutual respect began to emerge, but it was not a smooth process. In 1967, the Soviet spacecraft Venera 4 parachuted into Venus's atmosphere. It measured a pressure of 2,000 kilopascals and a temperature of $275^{\circ} \mathrm{C}$ before it stopped transmitting. Soviet scientists insisted that the probe had reached the planet's surface, although there was already evidence that temperatures and pressures there would be higher. A newly minted PhD at the time, I remember being struck by their

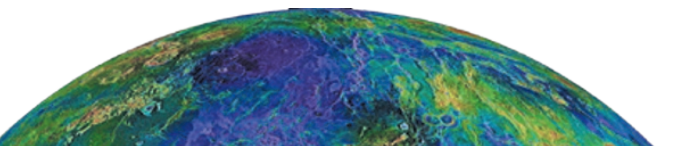

planetary bodies, and Venus's remaining mysteries. What maintains the planet's extraordinary greenhouse effect? If Venus once had an ocean, where is the water now? Why does the wind at the top of the clouds blow 50 times faster than on the ground, at more than twice the speed of Earth's jet streams? And why does Venus have an atmosphere of carbon dioxide almost 100 times more massive than Earth's nitrogen-oxygen atmosphere? For Venus's surface and interior, the big questions are: how does the planet shed its internal heat, given that there is no sign of plate tectonics? Are volcanoes enough? Are the volcanoes active and what gases do they bring to the surface? Is volcanic activity changing the climate of Venus? To answer some of these questions, space agencies are studying aeroplanes and rovers suitable for Venus. Operating on the planet's surface is difficult but not impossible: the instruments must either operate at high temperature, or be refrigerated.

The book reproduces numerous intriguing images of the surface taken by the radar on NASA's Magellan spacecraft. A chapter on the clouds, which are concentrated at 50-65 kilometres altitude (Earth's are at 0-15 kilometres) reveals much about the meteorology of the planet. Despite the stronger winds, the circulation of the atmosphere is smoother than Earth's - less interrupted by waves and eddies, with winds blowing more nearly east to west. Also, the massive atmosphere seems to have evened out the temperature difference between equator and pole. At the poles, the clouds mark the edges of a vortex that resembles the polar vortex on Earth.

Taylor clearly believes that Venus has a lot to tell us about our own planet - particularly through its greenhouse effect, winds and volcanoes, and the history of its surface. He writes wistfully of "the forgotten world" and the "unfulfilled objectives" of neverflown missions. The last part of the book is, however, forward-looking, covering plans and visions for further exploration of Earth's wayward next-door neighbour.

Andrew P. Ingersoll is professor of planetary science at the California Institute of Technology in Pasadena. He is the author of Planetary Climates. e-mail:api@gps.caltech.edu 